\title{
The Knees-Ankles-Toe in Cosmic Rays and the Periodic Table of Elementary Particles
}

\author{
Ding-Yu Chung \\ P. O. Box 180661, Utica, Michigan 48318, USA \\ Email: dy chung@yahoo.com
}

Received 18 June 2014; revised 15 July 2014; accepted 8 August 2014

Copyright (C) 2014 by author and Scientific Research Publishing Inc.

This work is licensed under the Creative Commons Attribution International License (CC BY). http://creativecommons.org/licenses/by/4.0/

(c) (7) Open Access

\section{Abstract}

This paper posits the discovery of the new elementary particles from the energy spectrum for the knees-ankles-toe of cosmic rays. The energy spectrum from $10^{9} \mathrm{eV}$ to $10^{20} \mathrm{eV}$ appears to follow a single power law except few breaks at the knees-ankles-toe. The power index increases at the first knee and the second knee, and decreases at the ankle. Above $4 \times 10^{19} \mathrm{eV}$, the power index increases as the "toe". The fine structure of the cosmic ray spectrum shows that an ankle with decrease in power index is in between the first knee and the second knee, resulting in two knees, two ankles, and one toe. This paper posits that the knees-ankles-toe are explained by the very high-energy fermions and bosons in the periodic table of elementary particles that places all known leptons, quarks, gauge bosons, and the Higgs boson in the table with the calculated masses in good agreement with observed values. In the periodic table, some high-energy dimensional fermions $\left(F_{d}\right.$ where $d=$ dimensional orbital number from 5 to 11$)$ and bosons $\left(B_{d}\right)$ are involved in the knees-ankles-toe. At the knees and the toe, some parts of the energies from the energy sources of cosmic rays are spent to generate $F_{d}$ and $B_{d}$, resulting in the increase of power index. The ankles are the the middle points (midpoints) between the adjacent dimensional fermions and bosons. At a midpoint, the energy is too high to keep the thermally unstable high-energy dimensional particle,resulting in the decay and the decrease of power index. The calculated masses of $B_{8}$, the midpoint, $F_{9}$, the midpoint, and $B_{9}$, are $1.7 \times 10^{15}, 2 \times 10^{16}, 2.4 \times 10^{17}, 2.8 \times 10^{18}$, and $3.2 \times 10^{19} \mathrm{eV}$, respectively, which are in good agreement with observed $3 \times 10^{15}, 2 \times 10^{16}, 3 \times 10^{17}, 3 \times 10^{18}$, and $4 \times 10^{19} \mathrm{eV}$ for the first knee, the first ankle, the second knee, the second ankle, and the toe, respectively. The mass of $F_{10}$ is $4.4 \times 10^{21} \mathrm{eV}$ beyond the GZK limit, so $F_{10}$ and above are not observed.

\section{Keywords}

Cosmic Rays, Knee, Ankle, Toe, the Periodic Table of Elementary Particles, New Elementary Particles 


\section{Introduction}

The study of cosmic rays has been important for the study of elementary particles. Before the advent of particle accelerators, subatomic particles such as muon and mesons were first discovered from cosmic rays. Currently, high-energy cosmic rays have much higher energies than the energy of particles accelerated by the Large Hadron Collider. Cosmic rays again attract great interest in the study of elementary particles beyond the capacity of particle accelerators. This paper posits the discovery of the new elementary particles from the energy spectrum for the knees-ankles-toe of cosmic rays.

The energy spectrum from $10^{9} \mathrm{eV}$ to $10^{20} \mathrm{eV}$ appears to follow a single power law $E^{-\gamma}$ except few breaks [1]. The power index, $\gamma$, increases at $3 \times 10^{15} \mathrm{eV}$ known as the "first knee", For energies above $3 \times 10^{17} \mathrm{eV}$, the spectrum is even steeper at the second knee. The power index decreases at $3 \times 10^{18} \mathrm{eV}$ known as the "ankle". The power index increases above $4 \times 10^{19} \mathrm{eV}$ as the "toe" [2]. The standard model for the origins of galactic cosmic rays is galactic supernova remnant (SNR). According to the SNR cosmic-ray hypothesis, charged particles are accelerated by the shock front created in a supernova and then further accelerated by the magnetic fields created in a supernova until they gain enough energy to escape this process and become newly formed cosmic rays. In the conventional theory, the knee marks the point where the magnetic fields as galactic accelerators reach their energetic limits, while the ankle marks the point where the galactic cosmic ray intensity falls below the intensity of cosmic rays from extragalactic sources of ultra-high-energy cosmic rays (UHECR) [3]. The exact reason for their presence is still an open question.

This paper posits that the knees-ankles-toe are explained by the very high energy fermions and bosons in the periodic table of elementary particles [4] [5] that places all known leptons, quarks, gauge bosons, and the Higgs boson in the table with the calculated masses in good agreement with observed values. In the periodic table, some high-energy dimensional fermions ( $F_{d}$ where $d=$ dimensional orbital number from 5 to 11$)$ and bosons $\left(B_{d}\right)$ are involved in the knees-ankles-toe. At the knees and the toe, some parts of the energies from the energy sources of cosmic rays are spent to generate $F_{d}$ and $B_{d}$, resulting in the increase of power index. The ankles are the middle points (midpoints) between the adjacent dimensional fermions and bosons. At a midpoint, the energy is too high to keep the thermally unstable high-energy dimensional particle, resulting in the decay and the decrease of power index. The calculated masses of the dimensional bosons, fermions, and the midpoints are in good agreement with the observed knees, ankles, and toe. The mass of $F_{10}$ is $4.4 \times 10^{21} \mathrm{eV}$ beyondthe GZK limit, so $F_{10}$ and above are not observed.

In Section 2, the cosmic rays for the knees-ankle-toe are discussed. Section 3 describes the periodic table of elementary particles. Section 4 deals with the knees-ankles-toe in the periodic table of elementary particles.

\section{The Cosmic Rays for the Knees-Ankles-Toe}

The fine structure of the cosmic ray spectrum measured in Tunka-133 [6] and KASCADE Grande [7] [8] experiments shows that the spectrum in the energy range of $10^{16}$ to $10^{18} \mathrm{eV}$ cannot be fitted with single power index. The power index decreases around $2 \times 10^{16} \mathrm{eV}$ for spectrum hardening and increases around $3 \times 10^{17} \mathrm{eV}$ for spectrum steepening. The decrease in power index at $2 \times 10^{16} \mathrm{eV}$ indicates the appearance of the "first ankle" before the "second ankle" at $3 \times 10^{18} \mathrm{eV}$ that also has a decrease in power index, and is usually considered as "the ankle". As a result, there are two knees, two ankles, and one toe. The first knee, the first knee, the first ankle, the second knee, the second ankle, and the toe are at $3 \times 10^{15} \mathrm{eV}, 2 \times 10^{16} \mathrm{eV}, 3 \times 10^{17} \mathrm{eV}, 3 \times 10^{18} \mathrm{eV}$, and $4 \times 10^{19} \mathrm{eV}$, respectively. Such spectrum signifies that the ankles with decrease in power index are the transition points between the two knees and between the second knee and the toe with increase in power index.

In terms of the sources of cosmic rays, cosmic rays below $10^{10} \mathrm{eV}$ are solar cosmic rays, and cosmic rays from $10^{10} \mathrm{eV}$ to $10^{18} \mathrm{eV}$ are galactic cosmic rays within the Milky Way. One viable explanation for the ultrahigh energy cosmic rays above $3 \times 10^{18} \mathrm{eV}$ with different chemical composition from the second knee is an extragalactic origin. The high energy extragalactic cosmic rays are derived from the diffusion of low energy protons $\left(<10^{17} \mathrm{eV}\right)$ on extragalactic magnetic fields [6].

\section{The Periodic Table of Elementary Particles}

In this paper, the knees-ankles-toe are explained by the very high energy fermions and bosons in the periodic table of elementary particles [4] [5] that places all known leptons, quarks, gauge bosons, and the Higgs boson in 
the table with the calculated masses in good agreement with observed values by using only four known constants: the number of the extra spatial dimensions in the eleven-dimensional membrane, the mass of electron, the mass of $Z$ boson, and the fine structure constant of electromagnetism. As the periodic table of elements based on the atomic orbitals, the periodic table of elementary particles is based on the seven mass dimensions as the seven dimensional orbitals from $d=5$ to $d=11$ derived from the extra space dimensions of eleven dimensional membrane as in Figure 1.

The seven dimensional orbitals are arranged as $F_{5} B_{5} F_{6} B_{6} F_{7} B_{7} F_{8} B_{8} F_{9} B_{9} F_{10} B_{10} F_{11} B_{11}$, where $F_{d}$ and $B_{d}$ are dimensional fermion and dimensional boson. The dimensional fermions below $F_{9}$ are leptons and quarks. To distinguish leptons and quarks, another set of seven dimensional orbitals is needed as the auxiliary dimensional orbitals between $d=7$ to $d=9$ in addition to the original principal dimensional orbitals. The principal dimensional orbitals are mainly for leptons, while the auxiliary dimensional orbitals are mainly for quarks as in Figure 2.

The previous communications [4] shows that the masses of fundamental particles are related to each other with three simple formulae, and that the use of accepted mass data allows calculation of masses of many other particles. The formulae are

$$
\begin{gathered}
M_{d, F}=M_{d, B} \alpha_{d, B}, \\
M_{d-1, B}=M_{d, F} \alpha_{d, F} \\
M_{d-1, B}=M_{d, B} \alpha_{d}^{2},
\end{gathered}
$$

where $\mathrm{d}$ is the dimensional orbital number from 6 to 11. Each dimension has its own $\alpha_{d}$, and all $\alpha_{d}$ 's except $\alpha_{7}\left(\alpha_{w}\right)$ of the seventh dimension (weak interaction) are equal to $\alpha$, the fine structure constant of electromagnetism. In the Standard Model, coupling constant as running coupling constant is based on the perturbative beta function where coupling constant changes logarithmically with energies. In Equation (1), different fermions and bosons with different $d$ 's have different starting masses and coupling constants based on the non-perturbative calculation for the starting masses and coupling constants from Equation (1). Each starting mass orcoupling constant then has its own running mass orcoupling constant based on the perturbative beta function. The Standard Model does not provide the starting masses of leptons, quarks, and gauge bosons, while the periodic table of elementary particles provides the starting masses of leptons, quarks, and gauge bosons by using Equation (1) and some other equations [4] [5]. Therefore, the periodic table of elementary particles is consistent with and supplementary to the Standard Model.

$$
\begin{array}{ll|l|l|l|l|l|l|l}
\mathrm{d}= & 5 & 6 & 7 & 8 & 9 & 10 & 11
\end{array}
$$

Figure 1. The seven mass dimensions as dimensional orbitals.

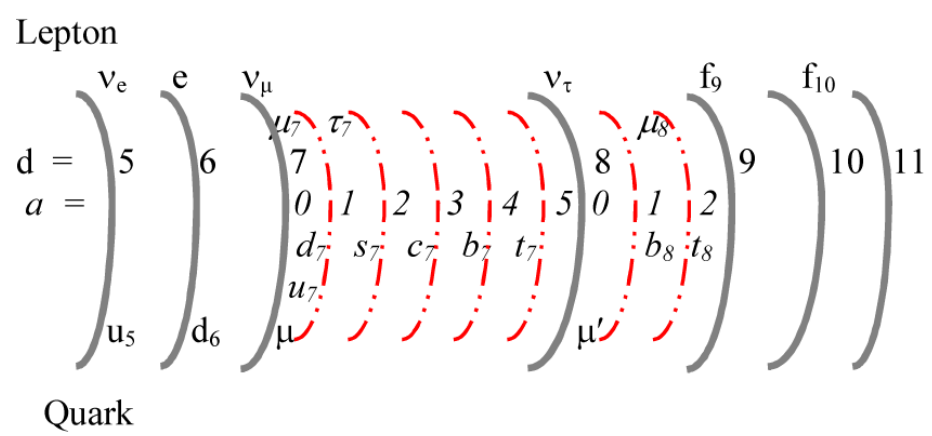

Figure 2. The seven principal dimensional orbitals (solid lines) denoted by the principal dimensional orbital number $d$ and the seven auxiliary dimensional orbitals (dash-dotted lines) denoted by the auxiliary dimensional number $a$. 
The periodic table with leptons, quarks, and gauge bosons is described in Reference [4] [5]. The periodic table of elementary particles without leptons and quarks is as Table 1.

In Table 1, $\alpha=\alpha_{e}$ (the fine structure constant for electromagnetic field), $M_{Z}=M_{w} / \cos \theta_{w}$, and $\alpha_{w}=\alpha / \sin ^{2} \theta_{w}$ [9]. $\alpha_{w}$ is not same as $\alpha_{e}$ because there is a mixing between $b_{5}$ and $b_{7}$ as the symmetry mixing between $\mathrm{U}(1)$ and $\mathrm{SU}(2)$ in the standard theory of the electroweak interaction, and $\sin \theta_{\mathrm{w}}$ is not equal to 1 . The calculated value for $\alpha_{w}$ is 0.02973 , and $\sin ^{2} \theta_{w}$ is 0.2454 in good agreement with 0.2312 for the observed value of $\sin ^{2} \theta_{w}$ [10].

The lowest energy boson $\left(B_{5}\right)$ at $d=5$ is the Coulomb field for electromagnetism. The second lowest boson $\left(B_{6}\right)$ at $d=6$ is $\pi_{1 / 2}$ (a spin 1 boson as a half of the spin 0 pion) for the strong interaction. The pion theory [11] where pions mediate the strong interaction works well at long enough distances (longer than the nucleon radius) or low enough energies. At short enough distances (shorter than the nucleon radius) or high enough energies, gluons emerge. In this paper, gluons are derived from the need to hide the auxiliary dimensional orbitals. As mentioned previously, leptons are derived mostly from the seven principal dimensional orbitals, while quarks are derived mostly from the seven auxiliary dimensional orbitals. Only the seven principal dimensional orbitals for leptons are revealed, while the seven auxiliary dimensional orbitals for quarks are hidden to preserve the seven apparent total dimensional orbitals instead of the fourteen apparent total dimensional orbitals. As a result, gluons emerge at the short distances to mediate the strong interaction among quarks in order to confine or hide isolated quarks (the auxiliary dimensional orbitals) within nucleons, while at the long distances without isolated quarks outside of nucleon at low energies, pions emerge to mediate the residual strong interaction for the nucleon-nucleon interaction or the nuclear force. (At low energies or long distances, gluons based on the negative perturbative beta function in QCD do not work due to the infrared divergence [12].) Although all of the gauge bosons in the Standard Model are originated from the local gauge symmetry, only gluon results in the confinement, while all other gauge bosons do not result in confinement. The confinement is not automatic for all gauge bosons, and only gluon results in the confinement.

The third lowest boson $\left(B_{7}\right)$ at $d=7$ is $Z^{0}$ for the weak interaction. In the Standard Model, $W^{ \pm}$bosons and $Z$ boson are related. The mass of $W$ bosons is equal to the mass of $Z$ boson times $\cos \theta_{w}$. Like photon at $d=5, Z$ is neutral, so $Z$ instead of $W^{ \pm}$is the dimensional boson at $d=7$. The highest energy boson $\left(B_{11}\right)$ is has the mass of the Planck mass for gravity. The calculated energy for $B_{11}$ is $1.1 \times 10^{28} \mathrm{eV}$ in good agreement with the Planck mass, $1.2 \times 10^{28} \mathrm{eV}$. The knees-ankles-toe in cosmic rays involves $B_{8}, F_{9}$, and $B_{9}$.

\section{The Knees-Ankles-Toe in the Periodic Table of Elementary Particles}

The paper posits that at knees and the toe, some parts of the energies from the energy sources of cosmic rays are

Table 1. The periodic table of elementary particles without leptons and quarks. $B_{\mathrm{d}}=$ dimensional boson, $F_{\mathrm{d}}=$ dimensional fermion, $M=$ mass $, \alpha=\alpha_{e}, \mathrm{~d}=$ dimensional orbital number.

\begin{tabular}{|c|c|c|c|c|c|}
\hline$B_{d}, F_{d}$ & $M_{d}$ & eV (calculated) & Force Fields & Observed Cosmic Ray & Main Source \\
\hline$B_{5}$ & $M_{e} \alpha$ & $3.7 \times 10^{3}$ & $\begin{array}{c}A=\text { photon } \\
\text { (electromagnetism) }\end{array}$ & & \\
\hline$B_{6}$ & $M_{e} / \alpha$ & $7 \times 10^{7}$ & $\pi_{1 / 2}$ (strong) & & solar \\
\hline$B_{7}$ & $M_{B 6} / \alpha_{w}^{2} \cos q_{w}$ & $9.1 \times 10^{10}$ (given) & $Z_{L}^{0}$ (weak) & & galactic SNR \\
\hline$B_{8}$ & $M_{B 7} / \alpha^{2}$ & $1.7 \times 10^{15}$ & & the first knee & galactic SNR \\
\hline$F_{9}$ & $M_{B 8} / \alpha$ & $2.4 \times 10^{17}$ & & the second knee & galactic SNR \\
\hline$B_{9}$ & $M_{F 9} / \alpha$ & $3.2 \times 10^{19}$ & & the toe & extragalactic UHECR \\
\hline$F_{10}$ & $M_{B 9} / \alpha$ & $4.4 \times 10^{21}$ & & beyond the GZK limit & not observed \\
\hline$B_{10}$ & $M_{F 10} / \alpha$ & $6.0 \times 10^{23}$ & & & \\
\hline$F_{11}$ & $M_{B 10} / \alpha$ & $8.3 \times 10^{25}$ & & & \\
\hline$B_{11}$ & $M_{F 11} / \alpha$ & $1.1 \times 10^{28}$ & Planck mass (gravity) & & \\
\hline
\end{tabular}


spent to generate $F_{d}$ and $B_{d}$, resulting in the increase of power index. The energy spectrum of cosmic rays is like the energy spectrum of a molecule. A slope change or peak in the curve of the spectrum of a molecule indicates the presence of a specific chemical bond, while aslope change or peak in the curve of the spectrum of cosmic rays indicates the presence of a specific dimensional particle. The ankles are the middle points (midpoints) between the adjacent dimensional fermions and bosons.

$$
M_{\text {midpoint }}=\exp \left(\left(\ln \left(M_{\text {adjacent dimensional ferion }}\right)+\ln \left(M_{\text {adjacent dimensional boson }}\right)\right) / 2\right)
$$

At a midpoint, the energy is too high to keep the thermally unstable high-energy dimensional particle, resulting in the decay and the decrease of power index. Unlike the thermally stable elementary particles for gravity and the Standard Model (the electroweak and the strong interactions), the high-energy dimensional particles outside of gravity and the Standard Model are not thermally stable. As a result, at the extremely high temperature in the beginning of the universe, only the thermally stable elementary particles for gravity and the Standard Model (the electroweak and the strong interactions) existed. The masses of leptons, quarks, and gauge bosons are too close together to be distinctive knees and ankles, so the knees-ankles-toe involves $B_{8}, F_{9}$, and $B_{9}$ whose masses are far apart to be detected as the knees-ankles-toe.

The calculated masses of $B_{8}$, the midpoint, $F_{9}$, the midpoint, and $B_{9}$, are $1.7 \times 10^{15}, 2 \times 10^{16}, 2.4 \times 10^{17}$, $2.8 \times 10^{18}$, and $3.2 \times 10^{19} \mathrm{eV}$, respectively, which are in good agreement with observed $3 \times 10^{15}, 2 \times 10^{16}, 3 \times$ $10^{17}, 3 \times 10^{18}$, and $4 \times 10^{19} \mathrm{eV}$ for the first knee, the first ankle, the second knee, the second ankle, and the toe, respectively as in Table 2 .

The mass of $F_{10}$ is $4.4 \times 10^{21} \mathrm{eV}$ beyondthe GZK (Greisen-Zatsepin-Kuzmin) limit, which occurs at about 5 $\times 10^{19} \mathrm{eV}$, as the maximum energy of cosmic ray particles that have traveled long distances (about 160 million light years), due to the theoretical energy losses of higher-energy ray particles and to scattering from photons in the cosmic microwave background. Therefore, $F_{10}$ and above are not observed.

\section{Summary}

This paper posits the discovery of the new elementary particles from the energy spectrum for the knees-ankles-toe of cosmic rays. The energy spectrum from $10^{9} \mathrm{eV}$ to $10^{20} \mathrm{eV}$ appears to follow a single power law except few breaks at the knees-ankles-toe. The power index increases at the first knee and the second knee, and decreases at the ankle. Above $4 \times 10^{19} \mathrm{eV}$, the power index increases as the "toe". The fine structure of the cosmic ray spectrum shows that an ankle with decrease in power index is in between the first knee and the second knee, resulting in two knees, two ankles, and one toe. This paper posits that the knees-ankles-toe are explained by the very high-energy fermions and bosons in the periodic table of elementary particles that places all known leptons, quarks, gauge bosons, and the Higgs boson in the table with the calculated masses in good agreement with observed values. In the periodic table, some high-energy dimensional fermions $\left(F_{d}\right.$ where $d=$ dimensional orbital number from 5 to 11$)$ and bosons $\left(B_{d}\right)$ are involved in the knees-ankles-toe. At the knees and the toe, some parts of the energies from the energy sources of cosmic rays are spent to generate $F_{d}$ and $B_{d}$, resulting in the increase of power index. The ankles are the middle points (midpoints) between the adjacent dimensional fermions and bosons. At a midpoint, the energy is too high to keep the thermally unstable highenergy dimensional particle, resulting in the decay and the decrease of power index. The calculated masses

Table 2. The calculated masses for dimensional bosons-fermionsand the observed eV for the knees-ankles-toe $B_{d}=$ dimensional boson, $F_{d}=$ dimensional fermion.

\begin{tabular}{ccccc}
\hline$B_{d}, F_{d}$ & Calculated eV & Cosmic rays & Observed eV \\
\hline$B_{8}$ & $1.7 \times 10^{15}$ & the first knee & $3 \times 10^{15}$ \\
the midpoint between $B_{8}$ and $F_{9}$ & $2 \times 10^{16}$ & the first ankle & $2 \times 10^{16}$ \\
$F_{9}$ & & $2.4 \times 10^{17}$ & the second knee & $3 \times 10^{17}$ \\
the midpoint between $F_{9}$ and $B_{9}$ & $2.8 \times 10^{18}$ & the second ankle & $4 \times 10^{18}$ \\
$B_{9}$ & & $3.2 \times 10^{19}$ & the toe & beyond the GZK limit \\
$F_{10}$ & $4.4 \times 10^{21}$ & not observed \\
\hline
\end{tabular}


of $B_{8}$, the midpoint, $F_{9}$, the midpoint, and $B_{9}$, are $1.7 \times 10^{15}, 2 \times 10^{16}, 2.4 \times 10^{17}, 2.8 \times 10^{18}$, and $3.2 \times 10^{19}$ $\mathrm{eV}$, respectively, which are in good agreement with observed $3 \times 10^{15}, 2 \times 10^{16}, 3 \times 10^{17}, 3 \times 10^{18}$, and $4 \times 10^{19}$ $\mathrm{eV}$ for the first knee, the first ankle, the second knee, the second ankle, and the toe, respectively. The mass of $F_{10}$ is $4.4 \times 10^{21} \mathrm{eV}$ beyond the GZK limit, so $F_{10}$ and above are not observed.

\section{References}

[1] Drury, L. (2012) Astroparticle Physics, 39-40, 52-60. http://dx.doi.org/10.1016/j.astropartphys.2012.02.006

[2] Watson, A. (2014) Reports on Progress in Physics, 77, Article ID: 036901. http://dx.doi.org/10.1088/0034-4885/77/3/036901

[3] Apel, W., et al. (2013) Physical Review D, 87, Article ID: 081101(R). http://dx.doi.org/10.1103/PhysRevD.87.081101

[4] Chung, D. (1997) Speculations in Science and Technology, 20, 259-268. http://dx.doi.org/10.1023/A:1026433207862

[5] Chung, D. and Hefferlin, R. (2013) Journal of Modern Physics, 4, 21-26. http://dx.doi.org/10.4236/jmp.2013.44A004

[6] Sveshnikova, L., Korosteleva, E., Kuzmichev, L, Prosin, V., Ptuskin, V. and Strelnikova, O. (2013) Journal of Physics: Conference Series (JPCS), 409, Article ID: 012062. http://dx.doi.org/10.1088/1742-6596/409/1/012062

[7] Apel, W., et al. (2012) Astroparticle Physics, 36, 183-194. http://dx.doi.org/10.1016/j.astropartphys.2012.05.023

[8] Apel, W., et al. (2011) Physical Review Letters, 107, Article ID: 171104. http://dx.doi.org/10.1103/PhysRevLett.107.171104

[9] Salam, A. (1968) Weak and Electromagnetic Interactions. In: Svartholm, W., Ed., Elementary Particle Theory, Almquist and Wiksell, Stockholm, 367-387.

[10] Beringer, J., et al. (2012) Physical Review D, 86, Article ID: 010001.8. http://pdg.lbl.gov/2012/reviews/rpp2012-rev-standard-model.pdf

[11] Fujita, J. and Miyazawa, H. (1957) Progress of Theoretical Physics, 17, 360. http://dx.doi.org/10.1143/PTP.17.360

[12] Aguilar, A., Binosi, D. and Papavassiliou, J. (2008) Physical Review D, 78, Article ID: 025010. 
Scientific Research Publishing (SCIRP) is one of the largest Open Access journal publishers. It is currently publishing more than 200 open access, online, peer-reviewed journals covering a wide range of academic disciplines. SCIRP serves the worldwide academic communities and contributes to the progress and application of science with its publication.

Other selected journals from SCIRP are listed as below. Submit your manuscript to us via either submit@scirp.org or Online Submission Portal.
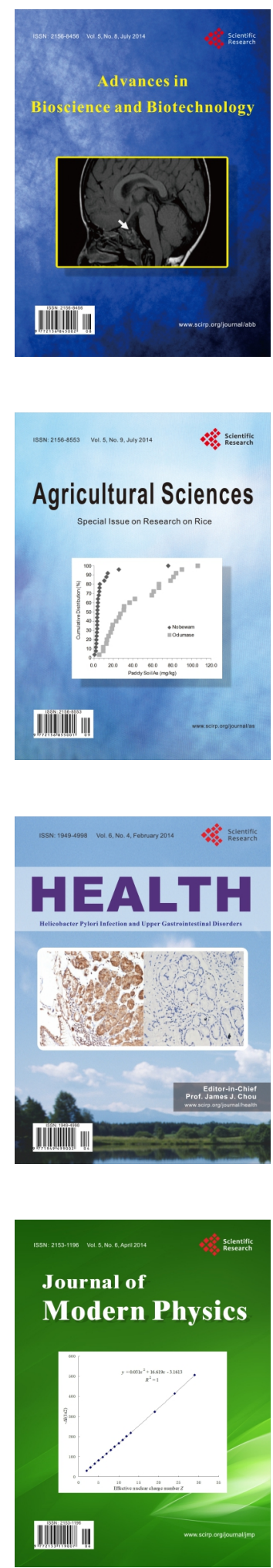
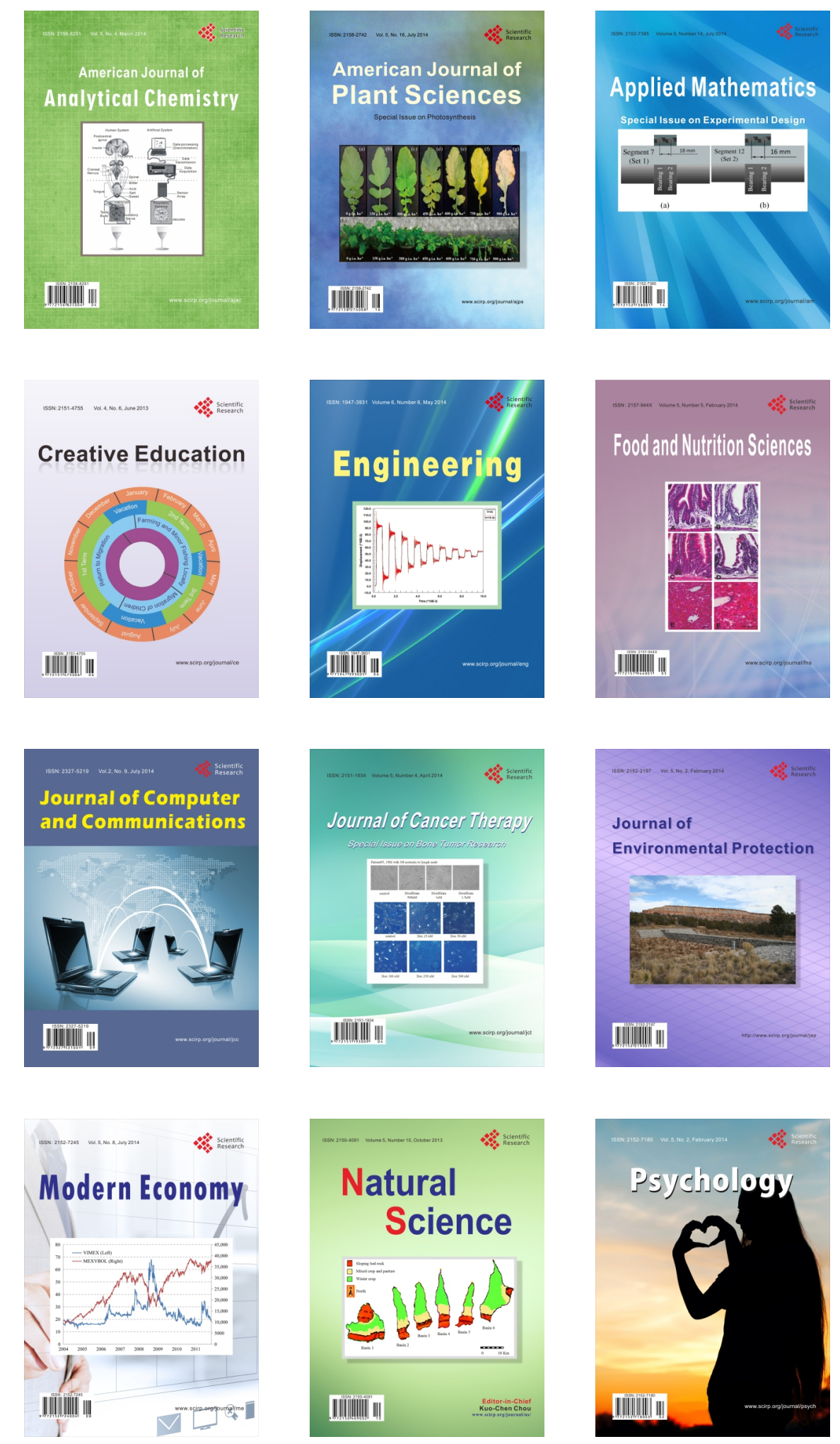